\title{
Prognostic significance of tumour stroma ratio in inflammatory breast cancer
}

\author{
Candice L Downey ${ }^{1}$, Helene H Thygesen ${ }^{2}$, Nisha Sharma ${ }^{1}$ and Abeer M Shaaban ${ }^{3^{*}}$
}

\begin{abstract}
Tumour stroma ratio (TSR) is emerging as an important prognostic indicator in cancer. We have previously shown TSR to be prognostic in oestrogen receptor positive breast cancer. Its role in inflammatory breast cancer, a rare but aggressive form of breast cancer, has not been identified. Here we aimed to determine the prognostic significance of TSR in a cohort of patients with inflammatory breast carcinoma.

TSR was measured by point counting virtual H\&E stained tissue sections in 45 inflammatory breast cancer cases. The whole tumour area was sampled. Optimum cut-offs to distinguish high and low TSR was determined by log-rank test. The relationship of TSR to overall survival and disease-free survival (DFS) was analysed alongside multivariate analysis.

The optimal cut-offs between high and low TSR were determined to be 31\% for OS and 46\% for DFS. There was no significant difference in OS ( $p=0.53)$ nor DFS $(p=0.66)$ between high and low TSR groups. Multivariate analysis did not demonstrate any new trends, within the limits of a small data sample. A significant correlation was found between pathological response to neoadjuvant chemotherapy and survival $(p=0.008)$.

There is no evidence that TSR has prognostic significance in inflammatory breast cancer. When compared with published data in non-inflammatory breast carcinoma, this supports the view that differences in stromal biology exist between tumour types and highlights the importance of considering this when interpreting the prognostic value of TSR. However, these findings must be interpreted in the light of the small sample size.
\end{abstract}

Keywords: Breast cancer; Inflammatory; Stroma; Tumour-stroma ratio; Prognosis

\section{Purpose}

Tumour-stromal ratio (TSR) is fast emerging as a significant prognostic indicator in cancer. The importance of TSR has been investigated in cancers including prostate (Yanagisawa et al. 2007), colorectal (West et al. 2010), oesophageal (Wang et al. 2012) and breast (de Kruijf et al. 2011) although results so far have been inconsistent.

Breast cancer is the most common cancer in the United Kingdom and the second most common cause of death from cancer in women after lung cancer (Office for National Statistics 2013). The vast heterogeneity of the disease necessitates multiple treatment options. Inflammatory breast cancer (IBC) is diagnosed clinically and it is characterized by rapid onset of diffuse erythema and warmth and oedema of the skin of the breast (peau

\footnotetext{
* Correspondence: Abeer.Shaaban@uhb.nhs.uk

${ }^{3}$ Department of Cellular Pathology, Queen Elizabeth Hospital Birmingham and University of Birmingham, Birmingham B15 2TW, UK

Full list of author information is available at the end of the article
}

d'orange). It is thought that emboli in lymphatic vessels cause their obstruction and prevents proper drainage of the lymph fluid thus causing swelling of the breast and its inflammatory-like appearance (Robertson et al. 2010). In 2011, an international panel published consensus criteria for the diagnosis of IBC (Dawood et al. 2011). More recently, a UK perspective on the diagnostic criteria, management, documentation and research directions has been proposed (Rea et al., Br J Cancer, in press).

Previous studies into TSR in breast cancer have largely focussed on hormone status, particularly triple-negative; that is, negative for ER, PR and HER2. Moorman et al. showed that a high percentage of stroma predicts poor survival in triple-negative breast cancers (Moorman et al. 2012). TSR was found to be an independent prognostic factor for relapse-free survival in breast cancer patients, and especially in those with triple-negative disease (de Kruijf et al. 2011). However, a study of

\section{实}


unselected breast cancer cases showed that TSR did not predict survival on multivariate analysis (Ahn et al. 2012). In addition, our previous study of ER-positive breast cancers demonstrated that a high TSR was related to worse survival across both genders (Downey et al. 2014). The prognostic role of TSR in inflammatory breast carcinoma has not been previously studied.

Here we aim to determine the prognostic significance of TSR in inflammatory breast cancer. The results will help to elucidate the scope of significance of TSR in breast cancer.

\section{Methods}

\section{Ethical approval}

Ethical approval for the study was granted by Leeds (East) Research Ethics Committee (Reference no: 06/ Q1206/180).

\section{Ethical standards}

All the work detailed herein was performed to comply with the current laws of the United Kingdom.

\section{Patients}

Patients with the diagnosis of inflammatory breast cancer between the period 2005-2013 were identified from the clinical database at the Leeds Teaching Hospitals NHS Trust. H\&E slides and paraffin-embedded tumour blocks of the original (pre-treatment) core biopsy were retrieved from the pathology archives. Comprehensive histological and clinical data including response to chemotherapy (complete, partial, minimal or no response), disease-free survival and overall survival were collected on those patients.

\section{Clinicopathological data}

Histopathological data was obtained from pathology databases. Relapse-free survival and overall survival was available for each patient.

\section{Measurement of stromal density}

Four $\mu \mathrm{m}$ thick haematoxylin and eosin-stained tissue sections were prepared according to standard protocols. Each slide was scanned at $20 \times$ magnification with an automated scanning system (Aperio XT, Aperio Technologies, Vista, CA, USA). Using a digital slide viewer (ImageScope version 8.0, Aperio Technologies), the whole area was selected for analysis. A grid with a systematic random sample of 300 points was then superimposed on the selected area using virtual graticule software (Treanor et al. 2008). The number of measurement points was consistent with that found accurate by previous studies (West et al. 2010). The histopathological category under each point was recorded and the number of points attributable to each
Table 1 Clinicopathological details of cohort

\begin{tabular}{|c|c|}
\hline Characteristic & (total $=45)$ \\
\hline Mean age (range) & $55(32-84)$ \\
\hline \multicolumn{2}{|l|}{ Type } \\
\hline Ductal & 33 (73\%) \\
\hline Lobular & $7(16 \%)$ \\
\hline Other & $5(11 \%)$ \\
\hline \multicolumn{2}{|l|}{ Grade } \\
\hline 1 & $3(7 \%)$ \\
\hline 2 & 20 (44\%) \\
\hline 3 & 22 (49\%) \\
\hline \multicolumn{2}{|l|}{$\mathrm{LN}$} \\
\hline No & $9(20 \%)$ \\
\hline N1-3 & 36 (80\%) \\
\hline \multicolumn{2}{|l|}{ ER } \\
\hline+ & 20 (44\%) \\
\hline- & $25(56 \%)$ \\
\hline \multicolumn{2}{|l|}{$P R$} \\
\hline+ & $11(24 \%)$ \\
\hline- & 34 (76\%) \\
\hline \multicolumn{2}{|l|}{ HER2 } \\
\hline+ & $13(29 \%)$ \\
\hline- & 32 (71\%) \\
\hline \multicolumn{2}{|l|}{ Size } \\
\hline$\leq 20 \mathrm{~mm}$ & 14 (31\%) \\
\hline$>20 \mathrm{~mm}$ & 29 (64\%) \\
\hline n/a & $2(4 \%)$ \\
\hline
\end{tabular}

category was counted. Categories used were tumour, stroma and non-informative (unclassifiable). Points falling on areas of lumen, necrosis, blood vessels, inflammation or blank areas fell into the latter category. TSR was expressed as a percentage of all the informative points per section.

\section{Statistical analyses}

Statistical analysis was performed using GraphPad Prism version 6, $R$ version 2.15 and the Survival package for $R$. The optimal cut-off value for TSR was calculated as follows: for each tumour proportion-value occurring in the data set, a log-rank test was performed based on a comparison of the group of patients with a TSR $\leq$ that value, and the patients with $\mathrm{P}$ above that. Primary endpoints

Table 2 Median OS and DFS between TSR groups

\begin{tabular}{lll}
\hline Median survival & High TSR & Low TSR \\
\hline OS & 75 & 35 \\
DFS & 57.5 & 37 \\
\hline
\end{tabular}




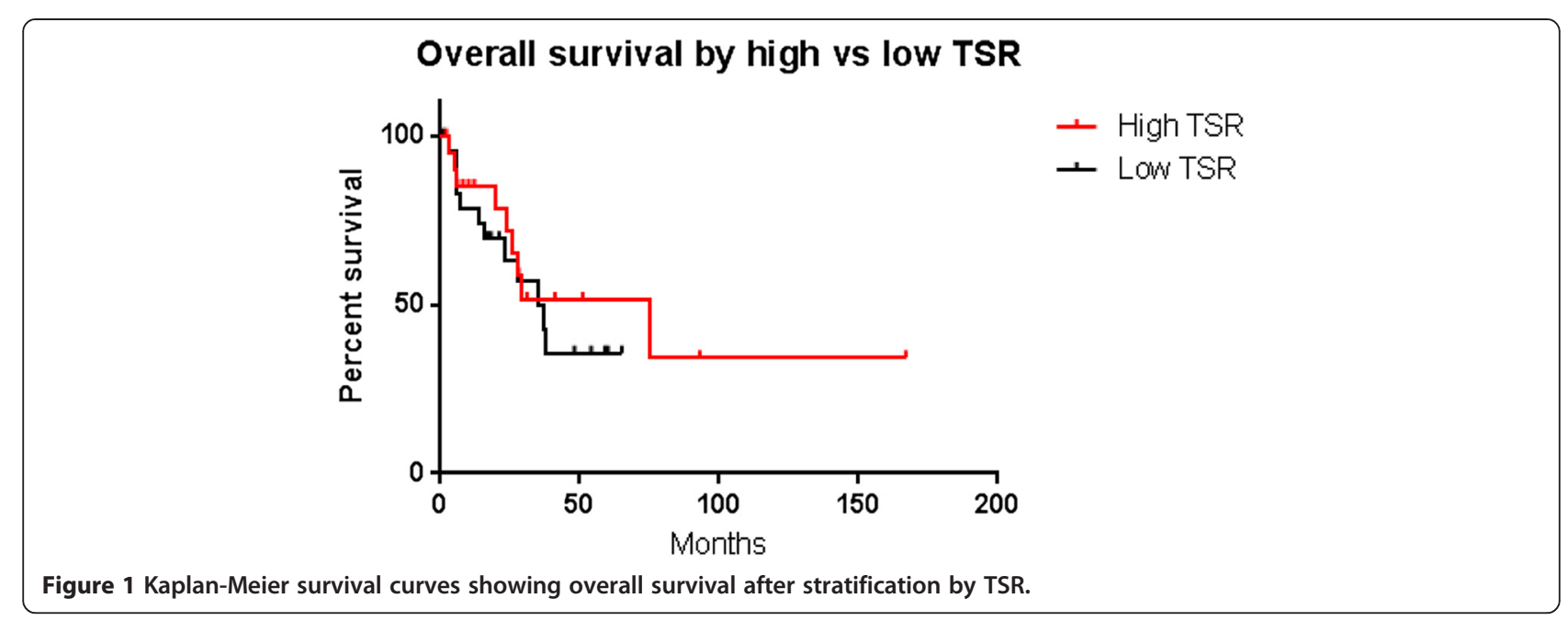

were disease-free survival (RFS) and overall survival. Univariate survival analyses were performed using Kaplan-Meier curves. Differences between the groups were assessed using the log-rank test. P-values of $<0.05$ were considered to be statistically significant. Multivariate analysis was performed using Cox's Proportional Hazards model.

\section{Results}

A total of 45 patients with available tumour slides/blocks were identified. The mean age at diagnosis was 55 years (+/- 12.8 years, range $32-84$ years).

Histopathological data obtained from pathology databases is summarised in Table 1. The majority of the tumours were of ductal no special type, node positive, hormone receptor and HER2 negative.

In order to determine optimum cut-offs to distinguish high and low TSR, log-rank tests were performed to compare groups of patients with TSR below or equal to that value, with patients with TSR above that. The cut- off values that led to the smallest $\mathrm{p}$-value were 0.31 for OS and 0.46 for DFS.

Median overall survival was 75 months in the high TSR group and 35 months in the low TSR group. A similar pattern was seen with the disease-free survival: 57.5 months in the high TSR group compared to 37 months in the low TSR group (Table 2).

There was no significant difference in survival between high and low TSR groups. This was true for both OS $(p=0.53$, Figure 1$)$ and DFS $(p=0.66$, Figure 2$)$.

Multivariate analysis demonstrated that pathological response (minimal or no response versus partial/ complete response) correlated with survival $(p=0.008)$ but TSR was not an independent prognostic factor for survival.

\section{Discussion}

The role of stromal microenvironment in dictating tumour behaviour has recently been emphasised. The initiation, growth and progression of cancer is dependent on

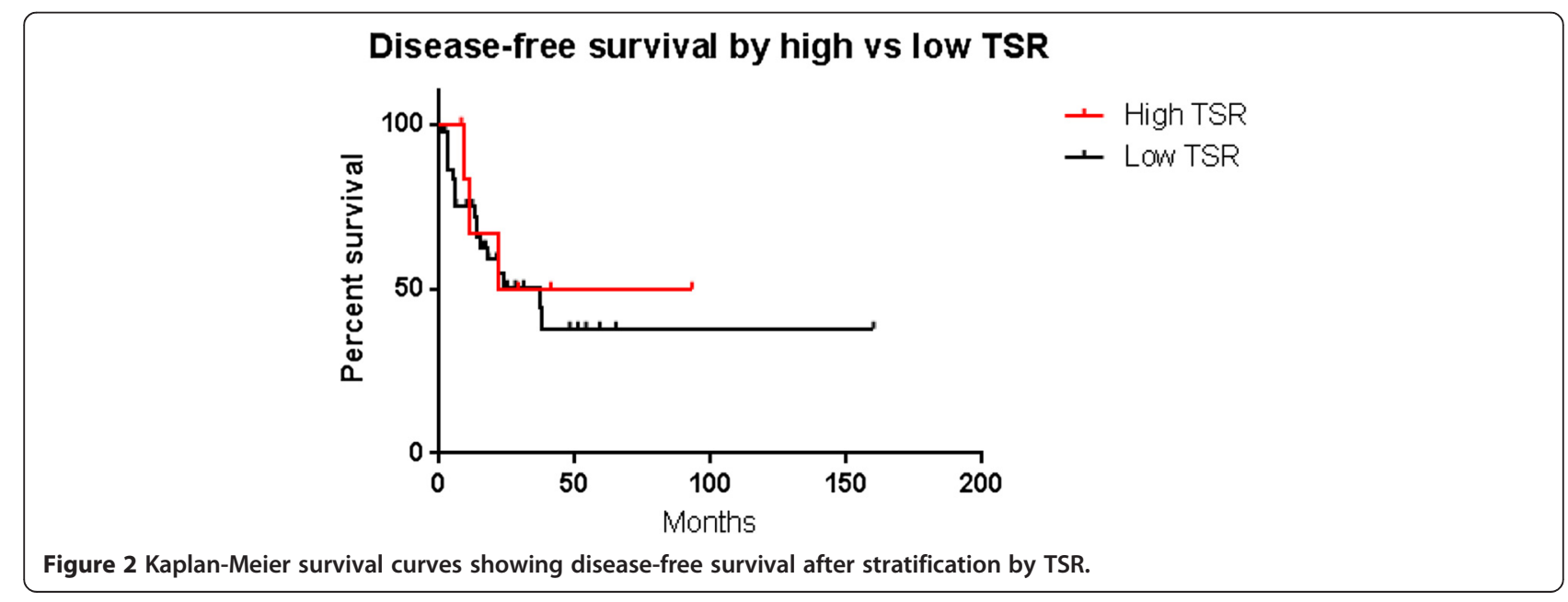


the tumour microenvironment of which tumour stroma is an integral part. Recently attention has focused on the potential prognostic value of TSR in different types of cancer. In general, low TSR appears to be associated with worse prognosis; in breast (de Kruijf et al. 2011); (Moorman et al. 2012); (Ahn et al. 2012); lung (Maeshima et al. 2002); prostate (Yanagisawa et al. 2007); stomach (Wu et al. 2013)and colon and rectum (West et al. 2010); (Mesker et al. 2007).

Current literature describes conflicting results, especially in breast, and seemingly dependent on hormone status. Whereas a number of studies on triple-negative breast cancers have found TSR to predict poor survival (de Kruijf et al. 2011; Moorman et al. 2012; Dekker et al. 2013), this prognostic value is diminished in studies of unselected breast cancers (Ahn et al. 2012). A study of ER-positive breast cancers demonstrated that a high TSR was related to worse survival across both genders (Downey et al. 2014) suggesting that the importance of TSR in breast cancer may be dependent on key molecular determinants of tumour subtype.

This is the first study to analyse stromal density and its relation to prognosis in inflammatory breast cancer. Our findings provide no evidence of any prognostic significance of TSR in inflammatory breast cancer. This finding adds to the literature base.

There are a number of methodological issues in this study. While there was a difference in survival between high and low TSR (75 months versus 35 months) suggestive of better outcome in the high stromal group, this did not reach statistical significance. Although 45 cases represent a relatively large cohort in this rare disease, the number of cases is small and the study is likely to be underpowered. The results need to be interpreted in this context.

Our findings suggest that differences in stromal biology may exist between tumour subtypes and highlights the importance of tumour subtype when interpreting the prognostic value of TSR. Future studies may elucidate crucial stromal differences between tumour subtypes which could better predict survival.

\section{Competing interests}

The authors declare that they have no competing interests.

\section{Authors' contributions}

CLD performed the analysis on digitised slides, recorded data and produced the first manuscript draft, HHT conducted the statistical analysis, NS identified cases and provided imaging data, AMS identified cases, provided histological sections, histological and follow up data, oversaw the analysis. All authors read and approved the final manuscript.

\section{Author details}

'St James's University Hospital, Leeds LS9 7TF, UK. ${ }^{2}$ Leeds Institute of Cancer \& Pathology, University of Leeds, St James's University Hospital, Leeds LS9 7TF, UK. ${ }^{3}$ Department of Cellular Pathology, Queen Elizabeth Hospital Birmingham and University of Birmingham, Birmingham B15 2TW, UK.

Received: 22 January 2015 Accepted: 23 January 2015

Published online: 10 February 2015

\section{References}

Ahn S, Cho J, Sung J, Lee JE, Nam SJ, Kim KM, Cho EY (2012) The prognostic significance of tumor-associated stroma in invasive breast carcinoma. Tumour Biol 33(5):1573-1580

Dawood S, Merajver SD, Viens P, Vermeulen PB, Swain SM, Buchholz TA, Dirix LY, Levine PH, Lucci A, Krishnamurthy S, Robertson FM, Woodward WA, Yang WT, Ueno NT, Cristofanilli M (2011) International expert panel on inflammatory breast cancer: consensus statement for standardized diagnosis and treatment. Ann Oncol 22(3):515-523

de Kruijf EM, van Nes JG, van de Velde CJ, Putter H, Smit VT, Liefers GJ, Kuppen PJ, Tollenaar RA, Mesker WE (2011) Tumor-stroma ratio in the primary tumor is a prognostic factor in early breast cancer patients, especially in triple-negative carcinoma patients. Breast Cancer Res Treat 125(3):687-696

Dekker TJ, van de Velde CJ, van Pelt GW, Kroep JR, Julien JP, Smit VT, Tollenaar RA, Mesker WE (2013) Prognostic significance of the tumor-stroma ratio: validation study in node-negative premenopausal breast cancer patients from the EORTC perioperative chemotherapy (POP) trial (10854). Breast Cancer Res Treat 139(2):371-379

Downey CL, Simpkins SA, White J, Holliday DL, Jones JL, Jordan LB, Kulka J, Pollock S, Rajan SS, Thygesen HH, Hanby AM, Speirs V (2014) The prognostic significance of tumour-stroma ratio in oestrogen receptor-positive breast cancer. Br J Cancer 110(7):1744-1747

Maeshima AM, Niki T, Maeshima A, Yamada T, Kondo H, Matsuno Y (2002) Modified scar grade: a prognostic indicator in small peripheral lung adenocarcinoma. Cancer 95(12):2546-2554

Mesker WE, Junggeburt JM, Szuhai K, de Heer P, Morreau H, Tanke HJ, Tollenaar RA (2007) The carcinoma-stromal ratio of colon carcinoma is an independent factor for survival compared to lymph node status and tumor stage. Cell Oncol 29(5):387-398

Moorman AM, Vink R, Heijmans HJ, van der Palen J, Kouwenhoven EA (2012) The prognostic value of tumour-stroma ratio in triple-negative breast cancer. Eur J Surg Oncol 38(4):307-313

Office for National Statistics (2013) Breast cancer incidence, mortality and survival, England, 1971-2011 Infographic Vol. No. 42, 2011 Release Part of Cancer Statistics Registrations, England (Series MB1)

Robertson FM, Bondy M, Yang W, Yamauchi H, Wiggins S, Kamrudin S, Krishnamurthy S, Le-Petross H, Bidaut L, Player AN, Barsky SH, Woodward WA, Buchholz T, Lucci A, Ueno NT, Cristofanilli M (2010) Inflammatory breast cancer: the disease, the biology, the treatment. CA Cancer I Clin 60(6):351-375

Treanor D, Dattani M, Quirke P, Grabsch H (2008) Systematic random sampling with virtual slides: a new software tool for tissue research 2008. J Pathol 216 (suppl 1):s43, Abstract

Wang K, Ma W, Wang J, Yu L, Zhang X, Wang Z, Tan B, Wang N, Bai B, Yang S, Liu H, Zhu S, Cheng Y (2012) Tumor-stroma ratio is an independent predictor for survival in esophageal squamous cell carcinoma. J Thorac Oncol 7 (9):1457-1461

West NP, Dattani M, McShane P, Hutchins G, Grabsch J, Mueller W, Treanor D, Quirke P, Grabsch H (2010) The proportion of tumour cells is an independent predictor for survival in colorectal cancer patients. Br J Cancer 102(10):1519-1523

Wu Y, Grabsch H, Ivanova T, Tan IB, Murray J, Ooi CH, Wright Al, West NP, Hutchins GG, Wu J, Lee M, Lee J, Koo JH, Yeoh KG, van Grieken N, Ylstra B, Rha SY, Ajani JA, Cheong JH, Noh SH, Lim KH, Boussioutas A, Lee JS, Tan P (2013) Comprehensive genomic meta-analysis identifies intra-tumoural stroma as a predictor of survival in patients with gastric cancer. Gut 62(8):1100-1111

Yanagisawa N, Li R, Rowley D, Liu H, Kadmon D, Miles BJ, Wheeler TM, Ayala GE (2007) Stromogenic prostatic carcinoma pattern (carcinomas with reactive stromal grade 3 ) in needle biopsies predicts biochemical recurrence-free survival in patients after radical prostatectomy. Hum Pathol 38(11):1611-1620 